\title{
Design on Test Paper Generating System for ESL Examination Based on Genetic Algorithm
}

\author{
Yanjia Xu \\ Teaching and Research Institute of Foreign Languages, Bohai University, Jinzhou, 121013, China \\ xuyonglin2008@163.com
}

Keywords: ESL examination; Test paper generating system; Mathematical model; Objective function; Genetic algorithm

\begin{abstract}
ESL examination, instead of TOEFL and IELTS to apply for American universities, are increasingly sought after by the majority of learners. Test paper generating system for ESL examination is designed in this paper to help teachers free from the onerous task of generating test paper. The efficiency and quality of test paper generating system depends on its algorithm design, therefore, algorithm design must be started firstly, including the establishment of mathematical model and objective function of generating test paper; then, on the basis of the steps of genetic algorithm, such as coding, selection, crossover and mutation, etc., elaborating the implementation process of test paper generating algorithm; and finally, according to the previous design, carried out the entire process design for test paper generating system. Test paper generating system based on genetic algorithm reduces the workload of teachers, so that proceducing papers is more reasonable and efficient, which has good performance and practicality.
\end{abstract}

\section{Introduction}

"ESL" is the abbreviation of "English as a Second Language", which means to learn "English as a Second Language". It is integrated into English society as the goal, but it is a communication tool as the supplement of native language in a non-English social environment. And all students through the ESL examination can not only improve their language skills, but also can in-depth understand social structure and cultures of the Anglo-American countries, so that their language skills can successfully achieve the level of life and learning in the United States [1,2]. English exam in the implementation process is not very scientific, test methods are backward, propositional content, examination scope, scoring criteria and methods are all affected by human factors, and it lacks of universal comparison, this unscientific test means reduces the effect and reliability of examination. With the advent and rapid development of the Internet age, test paper generating system has been widely used to complete the ESL exam.

Test paper generating technology is using a certain algorithm to make computer automatic extract corresponding number of questions from the specified test database to compose a test paper according to the requirements of teachers and teaching. In test systems, the quality of algorithm design on test paper generating directly affects the quality of the examination system, so the key for test system success is to select a scientific and efficient algorithm. Now commonly used test paper generating methods include random sampling method, recollection trial method and genetic algorithm and so on. The genetic algorithm is used in this paper to research.

Genetic algorithm is the computational model of simulating Darwinian' genetic selection and the natural elimination process of biological evolution, and is a method to search the optimal solution by simulating natural evolution process. It uses the law of nature that is "the survival of the fittest," through reproduction, crossover, mutation, and according to certain conditions to select a set of candidate solutions, finally selects optimum individuals from solution groups after multiple iterations [3]. 


\section{Algorithm Design on Test Paper Generating}

We all know that the ultimate goal of test paper is to generate an objective and scientific test papers with comprehensive knowledge. In order to achieve this goal, we need to complete the work of generating mathematical model and designing test paper algorithm. First we need to quantify various constraints of test paper, such as topic constraint, quantity constraint of topic, knowledge constraint, difficulty constraint and score constraint and so on, to a indicator system of test paper; then, using objective function that obtained by analyzing indicator system of the test paper to establish a mathematical model and solve it; finally to find questions with the same solution or the closest solutions in network test database to generate test paper and complete solving process of test paper. The solving process is the work of algorithm needs to be done [4].

(1) Mathematical model of generating test paper

According to the analysis, constraints of test paper include the following aspects essentially: total constraint, time constraint, topic constraint, score constraint in each section, knowledge constraint and difficulty constraint. Suppose that there are $n$ questions in a paper, the resulting paper $R$ can be represented by a $n \times 6$ matrix, namely:

$$
R=\left[\begin{array}{cccc}
a_{11} & a_{12} & \cdots & a_{16} \\
a_{21} & a_{22} & \cdots & a_{26} \\
\cdots & \cdots & \cdots & \cdots \\
a_{n 1} & a_{n 2} & \cdots & a_{n 6}
\end{array}\right]
$$

Wherein, every constraint in $R$ must meet the following requirements:

Total constraint: $A=\sum_{i=1}^{n} a_{i 1}$, wherein, $A$ represents the total of test paper which values 100 usually; $a_{i 1}$ represents the score of $i$ question.

Score constraint of each topic: $B_{t}=\sum_{i=1}^{n} a_{i 1} \times b_{1 i}$, wherein, $B_{t}$ represents the total scores of $t$ type of questions which is set by users usually, and test paper generally includes choice question, fill in the blank, true or false, short answer question and calculation question and so on; $b_{1 i}=\left\{\begin{array}{ll}1, & i \in t \\ 0, & i \notin t\end{array}\right.$.

Score constraint in each section: $C_{z}=\sum_{i=1}^{n} a_{i 1} \times b_{2 i}$, wherein, $C_{z}$ represents the total scores in $z$ section and usually sets $0 \leq C_{z} \leq 15 ; \quad b_{2 i}=\left\{\begin{array}{ll}1, & i \in Z \\ 0, & i \notin Z\end{array}\right.$.

Knowledge coverage constraint: $q=\frac{q^{\prime}}{Q}$, wherein, $q$ represents the actual knowledge coverage, $q^{\prime}$ represents the number of knowledge points in a test paper, $Q$ is the number of knowledge points that should be included in a paper; it general requires $q \geq 80 \%$.

Time constraint: $D=\sum_{i=1}^{n} a_{i 2}$, wherein, $D$ represents the total time completed test paper; $a_{i 2}$ represents the estimated time for $i$ question.

Difficulty constraint: $E=1-\frac{S_{a v g}}{S}$, wherein, $E$ represents the difficulty coefficient of a question; $S_{a v g}$ represents the average score of this question; $S$ represents the total score of this question.

(2) Objective function of generating test paper: 
According to the analysis for generating test paper procedures and generating test paper constraints, we can know that the question of generating test paper is to seek optimal solution in some constraints actually [5], so the objective function of generating test paper can be built. This paper obtains the sum of absolute value of the all deviations for the distributions, and finally the minimum sum is got to define the objective function of the problem. Suppose that each constraint corresponding function is $f(x)$, these components should be assigned a certain weight to indicate their importance when combine them, the mathematical model of multi-objective optimization established in this paper is as follows:

$$
\begin{aligned}
& f(x)=\left(f\left(x_{1}\right), f\left(x_{2}\right), f\left(x_{3}\right)\right) \\
& =\sum_{i=1}^{m}\left(B_{t}-B_{i}^{\prime} / B_{t}\right) * \omega_{1}+\sum_{i=1}^{o}\left(C_{z}-C_{i}^{\prime} / C_{z}\right) * \omega_{2}+\sum_{i=1}^{p}\left(E-E_{i}^{\prime} / E\right) * \omega_{3}
\end{aligned}
$$

Wherein, $\omega_{1}, \omega_{2}, \omega_{3}$ represent the weight of topic, section and difficulty, and $\sum_{\mathrm{i}=1}^{3} \omega_{\mathrm{i}}=1 ; m$ represents the number of topic, $B_{t}-B_{i}^{\prime}$ represents the difference between the actual score and required score of $i$-type questions; $o$ represents the total number of sections, $C_{z}-C_{i}^{\prime}$ represents the difference between the actual score and required score of $i$-section questions; $p$ represents the number of difficulty level, $E-E_{i}^{\prime}$ represents the difference between the actual score and required score of $i$-level questions. The three deviations mentioned above are smaller, the selected questions are closer to generating test paper requirements of the users; on the contrary, the deviations are larger, it means that the selected questions are farther from generating test paper requirements of the users. The ultimate goal of generating test paper is to obtain the minimum of the objective function, that is $\operatorname{minf}(x)$.

(3) Generating test paper process of genetic algorithm

Method of generating test paper based on genetic algorithm mainly includes the following steps: chromosome coding, generation of initial population, fitness function design, as well as through the genetic manipulation for the current group, such as selection, crossover and mutation, produces the next generation and gradually closes to the optimal status. Finally, design a termination condition. Specific test paper method as follows:

First, it is determined coding scheme. Using genetic algorithm to solve the problem, first solution space of the problem needs to be mapped into a code string. Literature [6] uses binary-encoding, 1 indicates that the question is selected, 0 indicates that the question is not selected, this coding is simple, but the number of all kinds of questions is difficult to control precisely when proceeds genetic manipulation. Moreover, the encoding is very long when the amount of questions in the database is large. So we use natural number coding. In the test paper design, we must first clear the number of questions in each topic, and make the question' score corresponding question number in each set of paper consistent with answer time, so that chromosome encoding can be classified according to topic. Suppose that $L$ questions met the constraints are selected from test database to generate a test paper, the encoding of $L$-length is the chromosome, each bit in it is represented by the question corresponding number, so that the coding numbers in chromosome are the numbers of questions in paper.

Second, the initial population is generated. Before genetic manipulation, we often start to generate the initial population and determine the size of the initial population firstly. While the system starts the initial search, in order to make each solution space with an equal chance, we generally in a random manner to generate the initial population. The size of population directly affects the performance and convergence speed of algorithm: if the fewer individuals involved in genetic manipulation are not too good for generating good model, however, too many individuals will reduce the performance and convergence speed of the algorithm. Usually user-defined method is used to set the size of the population. 
Third, it is determined the fitness function. Fitness function is used to quantify each chromosome, which is the key and core to achieve genetic algorithm. Fitness of each chromosome determines the pros and cons of the chromosome, and fitness values are calculated by the fitness function. Therefore we can say that the fitness function is the objective function of chromosome [7]. In the test paper algorithm, fitness function can be converted by the objective function, and fitness function used in this paper is as follows:

$$
F=1 / f(x)+1
$$

Wherein, $F$ is the fitness function, $f(x)$ represents the objective function as mentioned above. We can know from the formula (3), the fitness function is inversely proportional to the objective function, if the objective function is larger, the fitness function is smaller; on the contrary, the objective function is smaller, the fitness function is larger.

Fourth, the genetic manipulation is carried on.

Selection operation: selection operation of genetic algorithm should give priority to the best chromosome in the population, that is "the survival of the fittest" in genetic algorithm. Pros and cons of these chromosomes determine the probability of it being copied and retained in the next generation, large fitness value represents that it is the best chromosome with big chance of survival. This paper uses the method of roulette selection, first calculates the fitness of each individual, and accumulates to get $S_{j}$ in turn, finally gets $S_{n}$, wherein, $n$ represents the number of individuals, and then generate a random number $k$ between $\left[0, S_{n}\right]$, and compare $S_{j}$ and $k$ in turn, then $S_{j} \geq k$ represents that this individual is selected, finally repeat this step until the number of copies reach the goal;

crossover operation: crossover is that two individuals begin bit-by-bit exchange from a certain position in accordance with a certain probability (exchange probability is $p$ ). This paper uses single-point crossover in the same segment according to topic and multi-point crossover in the entire chromosome. On the one hand, it makes characteristics of excellent individuals in the original population can be maintained to some extent; on the other hand, it makes the algorithm to explore new gene space, so that individuals in the new group have diversity.

mutation operation: the problem of repeated question number or question number not existed may occurred after crossover operation, mutation operation is needed to use to select unused question number. Mutation is that select one individual in the population randomly firstly, and it is needed to change the value of a string in the string structure data for selected individual with a certain probability.

Fifth, design the termination condition. User requirements are not the same because of different populations, and therefore cannot be specified in the genetic algorithm that population evolution to which generation will be able to get the best individual, that is the best paper. First determine a threshold value, then calculate the fitness; if the fitness value is less than or equal to this threshold, the operation is end, if it is greater than the threshold, then continue mutation operation for other gene randomly, carry on heredity until fitness is less than or equal to this threshold, finally paper is generated [8].

\section{System Process Design}

Genetic algorithm is a development-oriented optimization algorithm of global search, it does not have calculation steps of regular pattern, we must appropriate adjust the steps of the algorithm based on specific issues in the application of genetic algorithms to solve practical problems. In solving practical problems, we must first design the entire process of the algorithm to have a clear understanding for concrete steps. Specific flow chart is shown in Fig. 1:

\section{Conclusion}


ESL examination is getting more and more people's attention, and the number of candidates is gradually increasing, so combining this situation, the paper designs generating test paper system to help ease the burden on the teachers of generating test paper to provide efficient exam service. Test paper is the multi-objective parameter optimization problem that needs to meet certain constraints and it belongs to NP-hard problem [9]. The design of generating test paper algorithm determines the efficiency and quality of generating test paper. Therefore, it is inevitable to research the generating test paper algorithm and it has important practical significance. This paper focuses on the characteristics of the generating test paper problem in actual system, and researches the application of genetic algorithm in generating test paper algorithm. The system can not only greatly reduce the cumbersome workload of artificial generating test paper, but also help to improve the quality of the test [10].



Fig. 1. Test paper logic diagram of genetic algorithm

\section{References}

[1] M. Zhang, "ESL teaching and suggestions on national preparatory English teaching," Journal of Language and Literature Studies, vol. 34, no. 6, pp. 81-82, 2014.

[2]

Baidu Encyclopedia,

"ESL," http://baike.baidu.com/link?url=-m8dHmQuIfDVpWaZbAc2mJI86pJmDsZPq8JcvAAH7HuJI HXBJBxVFvRINwAkw-EAEV0wKqGmS_F5pqdkqcmBBhZKMQxUtv_mIHyCNIZ4TBm, 2015-7-15.

[3] T. Xu, "Research and design on test paper generating based on genetic algorithm," Software Guide, vol. 13, no. 12, pp. 109-111, 2014.

[4] L. Pan, "Application method research of genetic algorithms in automatic generating test paper," Master's degree of Northeast Normal University, 2010.

[5] K. H. Cao, R. H. Gao, "Application of genetic algorithm in generating test paper system," Journal of Henan College of Finance \& Taxation, vol. 28, no. 6, pp. 76-78, 2014.

[6] X. J. Li, "Design and implementation of group volume system based on genetic algorithm," Modern Computer, vol. 30, no. 33, pp. 57-60, 2013.

[7] Y. Cao, "The design and implementation of intelligent online exam system based on genetic algorithm," Master's degree of University of Chinese Academy of Sciences, 2014.

[8] Z. Q. Wei, "Research and design of automatic generating test paper for adult education based on genetic algorithm," Journal of Guilin University of Aerospace Technology, vol. 20, no. 1, pp. 14-19, 2015. 
[9] J. Shi, Y. Miu, "Research and application of auto test paper generation based on genetic algorithm," Computer Knowledge and Technology, vol. 10, no. 34, pp. 8160-8162, 2014.

[10] Y. He, C. Q. Chen, "Research on technology of automatic paper generation based on genetic algorithm of mandarin test," Electronic Test, vol. 22, no. 4, pp. 31-32, 2014. 